\title{
ORIGINAL ARTICLE \\ Duration of the flaxseed diet promotes deposition of n-3 fatty acids in the meat and skin of Peking ducks
}

\author{
Muhammad Suhaib Shahid, Yuqin Wu, Zhibin Xiao, Tausif Raza, Xiaoyu Dong and \\ Jianmin Yuan* \\ State Key Laboratory of Animal Nutrition, College of Animal Science and Technology, China Agricultural University, \\ Beijing, China
}

\section{Popular scientific summary}

- Flaxseed was fed to ducks for 0, 10, 20, and 30 days.

- Flaxseed decreased growth performance and abdominal fat.

- A 30-d feeding improved meat quality and increased expression of Lipin-1 and FADS2 genes.

- Flaxseed fed over a period of 20 days enriched Peking duck's meat and skin with linolenic acid, DHA, and EPA, making it suitable for health conscious consumers.

Abstract

Background: Polyunsaturated fatty acids (PUFA), particularly n-3, have beneficial effects on human health, and for this reason foodstuffs with increased content of n-3 PUFA are now very common and widely available. Design: This study was conducted to investigate the effect of the duration of a flaxseed diet on Peking duck's growth performance, antioxidant status, gene expression, and fatty acid profile of the meat. A total of 792 12-day-old white Peking ducks were divided into four groups. In the control group, animals were provided with a basal diet. In the three experimental groups, animals were fed a 10\% flax seed diet with vitamin $\mathrm{E}$ at 13, 23, and 33 days of age for 30, 20, and 10 days, respectively.

Results: The growth performance of the ducks decreased with flaxseed diet's duration. Both body weight and body weight gain decreased linearly while Feed conversion ratios (FCR) increased in the group of ducks fed flaxseed compared to control ducks. Serum triglycerides (TG), very low density lipoprotein (VLDL), low density lipoprotein cholesterol (LDL-C), and aspartate aminotransferase (AST) linearly decreased while high density lipoprotein cholesterol (HDL-C) and lipopolysaccharide (LPS) levels increased by feeding flaxseed up to 30 days. The expression of lipin-1 gene (LPIN-1) and fatty acid desaturase 2 (FADS2) linearly increased in ducks fed flaxseed for 30 days. Linolenic acid (n-3) and its long-chain metabolites like eicosatetraenoic acid (ETA), eicosapentaenoic acid (EPA), docosahexaenoic acid (DHA), and total n-3 fatty acids (FA) linearly increased while the ratio of n-6 to n-3 was reduced with increased duration of flaxseed supplementation.

Conclusion: Overall, we found that increasing the duration of flaxseed diet with vitamin E for more than 10 days had a mild adverse effect on duck's growth performance but enrichedits meat with long-chain PUFA and decreased the n- 6 to n-3 ratio, providing quality meat for health-conscious consumers. A period of 20 days is good for producing n-3 enriched Peking duck meat and skin.

Keywords: flaxseed; LPIN-1; FADS2; DHA; meat; duck

I n humans, high ratios of $n-6 / n-3$ polyunsaturated fatty acids (PUFA) increase the risk of obesity and cardiovascular diseases (1). Because n-3 PUFA has beneficial effects on cardiovascular and neurological health, inflammation and immunity (2), it is possible to improve human nutrition by supplying $\mathrm{C} 18: 3 \mathrm{n}-3$ enriched foods (3). Chickens cannot have the capacity to synthesize n-3 PUFA, so these must be delivered in their feed.

Most lipids are ingested in the form of TG, but must be broken down into FA, and transported by fatty acidbinding proteins (FABP) or fatty acid transport protein 
(FATP) across the plasma membrane in the intestine $(4,5)$. In the liver, FA were metabolized by various genes like Lipin 1 (LPIN-1) and WD and tetra-Trico peptide repeats 1 (WDTC1) (6), n-3 FA were converted to DHA and EPA by FADS2 (7), and $\beta$-oxidized by the PPAR- $\alpha$ transcriptional factor in peroxisome and mitochondria (8). Previous studies reported that dietary n-3 increased the expression of FADS2 and LPIN1 genes in broilers $(6,9,10)$.

Lipoprotein lipase (LPL) takes part in the clearance of chylomicrons arising from dietary fat preventing it to enter the tissue and inhibiting TG accumulation (11). LPS is the part of the cell wall of Gram-negative bacteria that enter the circulation from the intestinal lumen in response to a high-fat diet (12).LPS decreased the LPL activity, thus interrupting the TG removal from circulation (13).

Flaxseed (Linum usitatissimum), is popular for its high content of n-3 and widely used as a source of n-3 (14). Recently, flaxseeds were also incorporated into food with other nutraceuticals to improve the nutritional quality of food (15). Studies on human and animals prove that consumption of flaxseed decreased the progression of atherosclerosis, decreased blood pressure, improved brain function, and provided protection against cancer (16). In poultry, it enhances the $\mathrm{n}-3$ content in eggs and meat, providing meat enriched in n-3 PUFA for which many positive health effects have been described (17). However, the presence of anti-nutritional factors like mucilage, linatinedi-peptide (a vitamin B6 antagonistic), cyanogenic glycosides, trypsin inhibitor, and phytic acid can limit its use in poultry feed (18). These anti-nutritional factors in flaxseed decreased body weight and increased the feed conversion ratio (FCR) in different species of birds $(19,20)$. In addition, feed enriched with PUFA can be prone to oxidation, causing oxidative stress in animals (21). A study showed that increasing the content of flaxseed up to $15 \%$ decreased the acceptability of broiler meat nuggets due to the lipid peroxidation of PUFAs (22). Previous studies also reported that flaxseed can enhance activities of ALT and AST (23). Therefore, antioxidants are added to diets of chickens to prevent oxidative damage (24). Combining n-3 FA with vitamin E could lower lipid oxidation activity and increase antioxidant status in plasma of broilers (25), without a negative impact on the physicochemical and sensory attributes of meat (19).

Roast Peking duck is a famous dish in the world. The skin comprises most of the roast Peking duck; however, it is normally enriched with n-6 FAs, which is not beneficial for people's health (26). Although many researchers studied the effect of duration of feeding flaxseed in broilers and pigs $(27,28)$, however, little attention was focused on the effect of the duration of flaxseed feed on Peking ducks. Therefore, the present study was conducted to investigate the effect of feeding period for flaxseed on performance, antioxidant status, $n-3$ content, and expression of genes related to fatty acid transport and metabolism of the Peking duck.

\section{Materials and methods}

All animal-related work was approved by the China Agricultural University Animal Care and Use Committee. All protocols and procedures were performed according to the Chinese Regulations for Laboratory Animals.

\section{Bird husbandry}

A total of 792 12-day-old white Peking ducks were divided into four groups. Every treatment group had six replicates with 33 ducks each. The control group (D) was given a basal diet from the 13th to the 42nd day. Groups on a flaxseed diet were provided a $10 \%$ flaxseed diet starting from the 13th, 23rd, and 33rd day of age for 30, 20, and 10 days, respectively. The composition of diets and nutrient analysis of diets and flaxseed are shown in Tables 1 and 2. Feed and water supply to the ducks were given ad-libitum.

\section{Measurements}

\section{Performance parameters}

Body weight (BW) and feed intake (FI) were recorded at the end of the experiment. Feed conversion ratios, survival rate, and European index were calculated.

Table 1. Ingredients composition of ducks diets

\begin{tabular}{lcc}
\hline Ingredient basis \% & Control diet & Flaxseed diet \\
\hline Corn & 65.50 & - \\
Wheat I3\% & - & 55.74 \\
$47 \%$ Soybean meal & 29.00 & 15.50 \\
Soy oil & 1.8 & - \\
Flaxseed & - & 10.00 \\
Barley & - & 15.00 \\
Calcium carbonate & 1.58 & 1.40 \\
Monocalcium phosphate 21\% & 1.30 & 1.30 \\
Salt & 0.36 & 0.36 \\
Methionine & 0.11 & 0.11 \\
Choline chloride, 60\% & 0.10 & 0.10 \\
Lysine HCl & 0.03 & 0.25 \\
Vitamin premix' & 0.02 & 0.02 \\
Vitamin E (50\%) & & 0.02 \\
Mineral premix ${ }^{2}$ & 0.20 & 0.20 \\
Total & 100.00 & 100.00 \\
\hline
\end{tabular}

'The vitamin premix provided the following per kilogram of diet: vitamin A, I $0,000 \mathrm{IU}$; vitamin $\mathrm{D}_{3}, 2,400 \mathrm{IU}$; vitamin $\mathrm{E}, 20 \mathrm{IU}$; vitamin $\mathrm{K}_{3}, 2.00 \mathrm{mg}$; thiamin, $2.00 \mathrm{mg}$; riboflavin, $6.40 \mathrm{mg}$; pyridoxine, $3.00 \mathrm{mg} ; \mathrm{VB}_{12}, 0.02 \mathrm{mg}$; folic acid, I.00 mg; pantothenic acid, $10.00 \mathrm{mg}$; nicotinic acid, $30.00 \mathrm{mg}$; biotin, $0.10 \mathrm{mg}$.

${ }^{2}$ The mineral premix provided following per $\mathrm{kg}$ of diet: $\mathrm{Cu}, 8 \mathrm{mg}$; Fe, 80 mg; Zn, 60 mg; Mn, 100 mg; I, 0.35 . 
Table 2. Nutrient analysis of duck diets

\begin{tabular}{lccc}
\hline Chemical composition & Control diet & Flaxseed diet & Flaxseed \\
\hline MEn Poultry (Kcal/kg) & 2950.0 & 2950.0 & \\
Protein \% & 19.0 & 19.0 & \\
Lysine \% & 1.01 & 1.01 & \\
Methionine \% & 0.40 & 0.40 & \\
M+C & 0.69 & 0.72 & \\
Threonine \% & 0.73 & 0.64 & \\
Ca\% & 0.90 & 0.90 & \\
Available phosphorus\% & 0.39 & 0.39 & \\
Fatty acids composition \% & Control diet & Flaxseed diet & \\
Myristic acid & 0.4 & 0.2 & 0.1 \\
Palmitic acid Cl6:0 & 9.8 & 8.4 & 6.7 \\
Margaric acid Cl7:0 & 0.3 & 0.2 & 0.1 \\
Palmitoleic acid Cl6:I & 0.12 & 0.09 & 0.08 \\
Oleic acid Cl8:In9c & 29.02 & 16.08 & 20.5 \\
Arachidic acid C20:0 & 0.14 & 0.08 & 0.09 \\
Linoleic acid Cl8:2n6 & 35.66 & 24.21 & 14.2 \\
Eicosadienoic acid & N.D & N.D & N.D \\
Dihomo- $\gamma$-linolenic acid & N.D & N.D & N.D \\
Alpha Linolenic acid n3 & 3.22 & 33.42 & 54.00 \\
ETA C20:3 n3 & N.D & N.D & N.D \\
EPA C20:5 n3 & N.D & N.D & N.D \\
DHA C22:6 n3 & N.D & N.D & N.D \\
\hline Note: & & & \\
\hline
\end{tabular}

Note: ETA = Eicosatetraenoic acid, EPA = Eicosapentaenoic Acid, $\mathrm{DHA}=$ docosahexaenoic acid, N.D = not detected.

\section{Sampling and preparation}

At the end of the experiment (day 30, when ducks' age was 42 days), one bird with a BW similar to the mean BW of the replicates in its group was selected for each treatment. A blood sample was collected from the wing's vein in a non-heparinized tube and centrifuged at 4,000 $\mathrm{g}$ for $15 \mathrm{~min}$. Serum was separated and stored at $-20^{\circ} \mathrm{C}$ for further analysis. After bleeding, birds were killed by stunning. Jejunum and liver were removed manually. A $0.5-\mathrm{cm}$ long section from the middle segment of the jejunum was collected from six birds per pen, flushed with cold PBS, and then frozen using liquid nitrogen and stored at $-80^{\circ} \mathrm{C}$ for further analysis.

\section{Carcass traits}

Carcass traits were measured according to Chinese performance terms and measurement method for poultry (29). Abdominal fat, subcutaneous fat, skin, and breast meat were removed manually from the carcass and weighted. Carcass traits such as skin, abdominal fat, and breast muscle were weighed. Their weights are expressed as relative weights (part weight/live weight) $\times 100$.

Meat quality

$\mathrm{pH}$ was measured $24 \mathrm{~h}$ post-mortem using a portable $\mathrm{pH} /{ }^{\circ} \mathrm{C}$ measuring instrument, Testo 206-pH2 and $\mathrm{pH} 2$ piercing probe head for semi-solid substances (Testo
GmbH \& Co., Lenzkirch, Germany). Drip loss from the breast meat was determined as described earlier (30).

\section{Serum biochemical and antioxidant indices}

The serum indices, namely TG, VLDL, LDL-C, HDL-C, alkaline phosphatase (ALP), AST, alanineamino transferase (ALT), LPL and LPS and were measured using commercially available kits (Nanjing Jiancheng Bioengineering Institute, China) following the manufacturer's instructions.

Determination of oxidative parameters in breast muscle, liver, and jejunal mucosa

The frozen breast muscle, jejunal mucosa, and liver pieces were homogenized in $0.86 \%$ (w/v) sodium chloride solution $\left(0.9 \mathrm{~mL}\right.$ added per gram of tissue) at $4^{\circ} \mathrm{C}$ using an U1tra-Turrax T8 homogenizer (IKA Labortechnik, Staufen, Germany) for 1-2 $\mathrm{min}$ at 3,000-5,000 r/min. The homogenates were centrifuged $\left(4,000 \mathrm{~g}\right.$ for $5 \mathrm{~min}$ at $\left.4^{\circ} \mathrm{C}\right)$ and the supernatants were used to determine the indices of oxidative stress. The oxidative indicators, SOD and MDA, in the breast muscle, liver, and jejunal mucosa were quantified using assay kits (Nanjing Jiancheng Bioengineering Institute, China) following the manufacturer's instructions (31).

\section{RNA extraction and reverse transcription}

Total RNA of liver samples was extracted using Trizol Reagent (Invitrogen Biotechnology Inc., Carlsbad, CA) according to the manufacturer's protocol. Sequences encoding the genes for duck $\Delta 6$-desaturase or FADS2, lipin1 gene (LPIN1), lipin 2 gene (LPIN2), L-FABP, peroxisome proliferator activated receptors alpha (PPAR- $\alpha$ ), WD and tetra-Trico peptide repeats 1(WDTC1), and FATP are shown in Table 3. Real-time PCR to measure the expression of lipid metabolism-related genes in the liver was carried out using SYBR Premix Ex Taq (TliRNaseH Plus) (Takara Biotechnology Inc., Osaka, Japan) on an ABI 7,500 real-time PCR System (Applied Biosystems, Foster City, CA). A reaction volume of $20 \mu \mathrm{L}$ of mixture contained $10 \mu \mathrm{L}$ SYBR Premix Ex Taq (TliRNaseH Plus) $(2 \times), 0.4 \mu \mathrm{L}$ ROX reference dye-II $(50 \times), 0.4 \mu \mathrm{L}$ each of forward and reverse primer (Table 2), $6.8 \mu \mathrm{L}$ of easy dilution, and $2 \mu \mathrm{L}$ of cDNA template. The optimized protocol for all the genes was $95^{\circ} \mathrm{C}$ for $30 \mathrm{~s}$ followed by 40 cycles of $95^{\circ} \mathrm{C}$ for $5 \mathrm{~s}$ and $60^{\circ} \mathrm{C}$ for $34 \mathrm{~s}$. All measurements were carried out in triplicate and the average values were obtained. Real-time PCR efficiency for each gene was calculated based on the slope of the cDNA relative standard curve that was formulated using a pooled sample. The specificity of the PCR products was evaluated by the analysis of the melting curve. Results of relative mRNA expression genes were calculated using the $2^{-\Delta \Delta \mathrm{Ct}}$ method (32).

\section{Fatty acid analysis}

For fatty acids (FA) analysis, $100 \mathrm{mg}$ of feed, minced meat, and skin were weighed and then extracted, saponified, and 
Table 3. Sequences of primer pairs of mRNA

\begin{tabular}{lll}
\hline mRNA & FORWARD & REVERSE \\
\hline LPIN I & TCATCCAGAGTGCTCCTGTG & TCTGGCTTATTTGCCTCAGC \\
LPIN 2 & CAGGATGGCACATACCAGTG & TCCATTGTCACCCAGTTTCA \\
FADS2 & TCTCCTCCTTGCATTCATCC & ACTTGTGGACGAGGTGGTTC \\
FATP & TCGCAGTGTATGGAGTGGAA & GGAGGCAGCACCTTCTGTAG \\
L-FABP & CACTGCCCCCACACTGCGTT & CGTCACCACAAAGTCGTCTCCT \\
PPAR- $\alpha$ & CAGAGTCATCCTTGCAGG & GTCAAGATTGGAGAAGCC \\
WDTCI & TATCAACGCAGCCCTCTTCT & CCTCAGCACCATCTCATCCT \\
$\beta$-actin & AAATCAAGATCATTGCCCCACCT & AGGGGTGTGGGTGTTGGTAA \\
\hline
\end{tabular}

Note: LPIN I: lipin I gene encoding lipin I protein; LPIN 2: lipin 2 gene encoding lipin 2 protein; FADS2:fatty acid desaturase 2 encoding $\Delta-6$ desaturase; FATP: fatty acid transport protein; FABP: fatty acidbinding protein; PPAR- $\alpha$ : peroxisome proliferatoractivated receptors alpha;WDTCI:WD and tetratricopeptide repeats I.

Table 4. Effect of supplemental different days of flaxseed on performances of Peking ducks

\begin{tabular}{|c|c|c|c|c|c|c|}
\hline Supplemental Days & $\mathrm{BW}(\mathrm{kg})$ mean $\pm \mathrm{SE}$ & $\mathrm{FI}(\mathrm{kg})$ & FCR & SR (\%) & $\mathrm{El}$ & BWG (kg) \\
\hline 30 & $3.38 \pm 0.0 \mathrm{I}^{\mathrm{b}}$ & $6.00 \pm 0.10$ & $2.67 \pm 0.02^{\mathrm{a}}$ & $97.48 \pm 0.93$ & $413.20 \pm 3.13^{b}$ & $2.33 \pm 0.0 \mathrm{I}^{\mathrm{C}}$ \\
\hline 20 & $3.39 \pm 0.04^{b}$ & $6.00 \pm 0.13$ & $2.60 \pm 0.0 \mathrm{I}^{\mathrm{a}}$ & $100.00 \pm 0.00$ & $427.00 \pm 7.32$ & $2.33 \pm 0.04^{c}$ \\
\hline 10 & $3.54 \pm 0.03^{\mathrm{a}}$ & $5.89 \pm 0.14$ & $2.4 \mathrm{I} \pm 0.03^{\mathrm{b}}$ & $97.48 \pm 0.93$ & $482.11 \pm 9.76^{a}$ & $2.50 \pm 0.03^{b}$ \\
\hline 0 & $3.61 \pm 0.02^{\mathrm{a}}$ & $5.72 \pm 0.05$ & $2.26 \pm 0.02^{c}$ & $97.98 \pm 0.64$ & $530.50 \pm 6.78^{a}$ & $2.57 \pm 0.12^{\mathrm{a}}$ \\
\hline \multicolumn{7}{|l|}{$P$ value } \\
\hline ANOVA & $<0.001$ & 0.215 & $<0.001$ & 0.073 & $<0.001$ & $<0.001$ \\
\hline LINEAR & $<0.001$ & 0.061 & $<0.001$ & $0.76 \mathrm{I}$ & $<0.001$ & $<0.001$ \\
\hline QUADRATIC & 0.305 & 0.368 & 0.003 & 0.183 & 0.026 & 0.237 \\
\hline
\end{tabular}

Note: $\mathrm{A}=30$ days, $\mathrm{B}=20$ days, $\mathrm{C}=10$ days, $\mathrm{D}=0$ days; data presented are mean; $n=6$; means in the same column are significantly different at $\alpha=0.05$; $\mathrm{BW}=$ body weight, $\mathrm{FI}=$ feed intake, $\mathrm{FCR}=$ feed conversion ratio, $\mathrm{SR}=$ survival rate, $\mathrm{EI}=$ European Index, $\mathrm{BWG}=$ body weight gain .

methylated according to a previously reported procedure of (33). The FA methyl esters were subjected to gas chromatography mass spectrometry (SCION-456, China) for fatty acid separation. The column measurements were $(30 \mathrm{~m} \times 0.32 \mathrm{~mm} \times 0.25 \mathrm{~m})$. The carrier gas used was Helium at a flow rate of $1.5 \mathrm{~cm}^{3} / \mathrm{min}$. The column, detector and injector temperatures were set at 195, 250, and $225^{\circ} \mathrm{C}$, respectively. FAs were identified comparing their retention times with authentic standards.

\section{Statistical analysis}

Data were analyzed as a completely randomized design using SPSS 20.0 (34). Linear and quadratic relations were used to check the significance. Post hoc significance was set at $P<0.05$.

\section{Results}

\section{Performance}

Feeding period of flaxseed linearly decreased body weight, FCR, European index, and body weight gain $(P<0.05)$ (Table 4). FI and survival rate were not significantly affected. Body weight decreased by $1.99,5.91$, and $6.16 \%$ for ducks fed flaxseed for 10,20 , and 30 days $(P<0.05)$, respectively and body weight gain decreased by $2.61,9.24$, and $9.04 \%$ for ducks fed flaxseed for 10, 20, and 30 days as compared to control ducks $(P<0.05)$. Feed conversion rate increased by $6.64,15.04$, and $18.14 \%$ in ducks fed flaxseed for 10,20 , and 30 days as compared to control ducks $(P<0.05)$.

\section{Carcass characteristics}

The relative weights of the skin, breast muscle, and abdominal fat were significantly affected by the duration of the flaxseed diet $(P<0.05)$ (Table 5). There were linear correlations between the duration of the flaxseed diet and the relative weight of skin and abdominal fat. Breast muscle's relative weight was quadratically affected by flaxseed duration $(P<0.05)$. Abdominal fat decreased linearly by 10.4 , 25.6, and $34.4 \%(P<0.05)$ in ducks fed flaxseed for 10,20 , and 30 days, respectively, as compared to control ducks.

\section{Quality of breast muscle}

Drip loss was significantly affected by flaxseed duration $(P<0.05)$ (Table 5). Drip loss of breast meat decreased linearly by $3.22,4.03$, and $32.66 \%$ for ducks fed flaxseed for 10,20 , and 30 days compared to the control $(P<0.05)$. The $\mathrm{pH}$ of breast meat was not significantly affected by duration of the flaxseed diet $(P<0.05)$. 


\section{Anti-oxidation character}

Breast muscle, liver, and jejunal mucosa SOD activity were not significantly affected by duration of the flaxseed diet $(P<0.05)$ (Table 6). However, MDA decreased linearly with duration of the flaxseed diet in the breast muscle, liver, and jejunal mucosa $(P<0.05)$.

\section{Fatty acids metabolic and endocrine hormone}

Serum contents of TG, VLDL, LDL-C, HDL-C, and activity of LPL, AST, LPS were significantly affected by duration of the flaxseed diet, while the activity of ALT and ALP were not significantly affected $(P<0.05)$ (Table 6). Serum HDL-C, LPL, and LPS increased linearly from 0 to 30 days of feeding flaxseed while TG, VLDL, LDL-C, and AST decreased linearly with increased duration of the flaxseed diet $(P<0.05)$.

\section{Gene expression in liver}

The expressions of hepatic genes like WDTC1, PPAR- $\alpha$, FATP, L-FABP, and LPIN2 were not significantly affected by duration of the flaxseed $\operatorname{diet}(P<0.05)$. Only the expressions of LPIN1 and FADS2 were found to linearly increase in ducks depending on the flaxseed duration $(P<0.05)$ (Table 7).

Fatty acid profile of breast muscle and skin

Both in the breast muscle (Table 8) and skin (Table 9), my ristic acid (C14:0), palmitic acid (C16:0), margaric acid

Table 5. Effect of supplemental different days of flaxseed on carcass traits and meat quality of Peking ducks

\begin{tabular}{|c|c|c|c|c|c|}
\hline \multirow[t]{2}{*}{ Supplemental days } & \multicolumn{3}{|c|}{ Relative weight (part weight/live weight $\times 100$ ) } & \multicolumn{2}{|c|}{ Meat quality } \\
\hline & Skin & Breast meat & A.Fat & $\mathrm{pH}$ & Drip loss (\%) \\
\hline 30 & $22.28 \pm 0.5 \mathrm{I}^{\mathrm{c}}$ & $8.37 \pm 0.35^{c}$ & $0.82 \pm 0.04^{b}$ & $5.61 \pm 0.02$ & $\mathrm{I} .67 \pm 0.2 \mathrm{I}^{\mathrm{b}}$ \\
\hline 20 & $23.55 \pm 0.63^{b}$ & $10.70 \pm 0.35^{\mathrm{a}}$ & $0.93 \pm 0.04^{b}$ & $5.63 \pm 0.04$ & $2.38 \pm 0.20^{\mathrm{a}}$ \\
\hline 10 & $25.60 \pm 0.64^{b}$ & $9.61 \pm 0.10^{b}$ & $1.12 \pm 0.09^{a}$ & $5.61 \pm 0.01$ & $2.40 \pm 0.30^{\mathrm{a}}$ \\
\hline 0 & $26.23 \pm 0.33^{\mathrm{a}}$ & $7.97 \pm 0.60^{c}$ & $1.25 \pm 0.05^{\mathrm{a}}$ & $5.64 \pm 0.02$ & $2.48 \pm 0.12^{\mathrm{a}}$ \\
\hline \multicolumn{6}{|l|}{$P$ value } \\
\hline ANOVA & 0.016 & 0.014 & 0.001 & 0.840 & 0.045 \\
\hline LINEAR & 0.002 & 0.388 & 0.001 & 0.499 & 0.017 \\
\hline QUADRATIC & 0.760 & 0.003 & 0.938 & 0.945 & 0.150 \\
\hline
\end{tabular}

Note: $\mathrm{A}=30$ days, $\mathrm{B}=20$ days, $\mathrm{C}=10$ days, $\mathrm{D}=0$ days; $\mathrm{A}$.Fat $=$ abdominal fat; data presented as mean; $n=6 ;$ means in the same column are significantly different at $\alpha=0.05$.

Table 6. Effect of supplemental different days of flaxseed on fatty acid metabolism, endocrine hormones and oxidative indices of Peking ducks

\begin{tabular}{|c|c|c|c|c|c|c|c|c|}
\hline & Supplemental days & 30 & 20 & 10 & 0 & $P$ & Linear & Quadratic \\
\hline \multirow[t]{9}{*}{ Serum } & TG (mmol/L) & $0.69 \pm 0.06^{b}$ & $0.76 \pm 0.07^{b}$ & $1.23 \pm 0.19^{a}$ & $1.44 \pm 0.2 \mathrm{I}^{\mathrm{a}}$ & 0.005 & 0.001 & 0.653 \\
\hline & LDL-C (mmol/L) & $1.92 \pm 0.05^{c}$ & $2.23 \pm 0.03^{b}$ & $2.47 \pm 0.05^{\mathrm{a}}$ & $2.50 \pm 0.03^{\mathrm{a}}$ & $<0.001$ & $<0.001$ & 0.003 \\
\hline & VLDL-C (mmol/L) & $1.03 \pm 0.0 \mathrm{I}^{\mathrm{d}}$ & $1.17 \pm 0.03^{c}$ & $1.31 \pm 0.03^{b}$ & $1.59 \pm 0.04^{\mathrm{a}}$ & $<0.001$ & $<0.001$ & 0.038 \\
\hline & HDL-C (mmol/L) & $1.48 \pm 0.03^{\mathrm{a}}$ & $1.36 \pm 0.02^{\mathrm{b}}$ & $1.24 \pm 0.03^{c}$ & $1.06 \pm 0.06^{d}$ & $<0.001$ & $<0.001$ & 0.448 \\
\hline & LPS (U/L) & $60.16 \pm 4.0 \mathrm{I}^{\mathrm{a}}$ & $50.53 \pm 3.27^{b}$ & $53.25 \pm 1.30^{\mathrm{ab}}$ & $37.56 \pm 1.60^{c}$ & $<0.001$ & $<0.001$ & 0.289 \\
\hline & LPL (U/mL) & $3.07 \pm 0.05^{\mathrm{a}}$ & $2.77 \pm 0.19^{a}$ & $2.47 \pm 0.28^{a}$ & $1.49 \pm 0.26^{\mathrm{b}}$ & $<0.001$ & $<0.001$ & 0.127 \\
\hline & ALP (U/L) & $1112.60 \pm 62.24$ & $1055.68 \pm 96.14$ & $1130.75 \pm 1.16$ & $1195.24 \pm 84.95$ & 0.761 & 0.442 & 0.517 \\
\hline & ALT (U/L) & $19.48 \pm 1.67$ & $18.38 \pm 1.48$ & $18.65 \pm 0.97$ & $19.58 \pm 1.55$ & 0.914 & 0.931 & 0.489 \\
\hline & AST (U/L) & $52.46 \pm 1.48^{b}$ & $69.33 \pm 18.03^{\mathrm{ab}}$ & $38.49 \pm 4.18^{b}$ & $105.25 \pm 17.50^{\mathrm{a}}$ & 0.009 & 0.037 & 0.065 \\
\hline \multirow[t]{2}{*}{ Meat } & MDA (nmol/mgprot) & $3.7 \mathrm{I} \pm 0.0 \mathrm{I}^{\mathrm{c}}$ & $3.72 \pm 0.0 \mathrm{I}^{\mathrm{c}}$ & $3.87 \pm 0.03^{b}$ & $4.35 \pm 0.03^{\mathrm{a}}$ & $<0.001$ & $<0.001$ & $<0.001$ \\
\hline & SOD (nmol/mgprot) & $210.93 \pm 0.38$ & $211.78 \pm 0.79$ & $210.33 \pm 1.19$ & $209.93 \pm 0.42$ & 0.369 & 0.209 & 0.424 \\
\hline \multirow[t]{2}{*}{ Liver } & MDA (nmol/mgprot) & $3.30 \pm 0.20^{b}$ & $3.53 \pm 0.25^{b}$ & $3.61 \pm 0.60^{b}$ & $5.28 \pm 0.28^{\mathrm{a}}$ & 0.004 & 0.002 & 0.067 \\
\hline & SOD (nmol/mgprot) & $209.98 \pm 19.25$ & $216.69 \pm 12.15$ & $198.66 \pm 7.45$ & $|8| .0 \mid \pm 4.55$ & 0.212 & 0.069 & 0.330 \\
\hline \multirow[t]{2}{*}{ Jejunum mucosa } & MDA (nmol/mgprot) & $3.46 \pm 0.17^{b}$ & $3.67 \pm 0.18^{b}$ & $3.95 \pm 0.20^{b}$ & $4.68 \pm 0.13^{\mathrm{a}}$ & $<0.001$ & $<0.001$ & 0.141 \\
\hline & SOD (nmol/mgprot) & $211.67 \pm 7.02$ & $221.11 \pm 9.90$ & $198.25 \pm 11.67$ & $199.44 \pm 6.76$ & 0.264 & 0.158 & 0.654 \\
\hline
\end{tabular}

Note: $\mathrm{A}=30$ days, $\mathrm{B}=20$ days, $\mathrm{C}=10$ days, $\mathrm{D}=0$ days. $\mathrm{TG}=$ triglycerides, $\mathrm{LDL}-\mathrm{C}=$ low density lipoprotein cholesterol, VLDL-C = very low density lipoprotein cholesterol, HDL-C = high density lipoprotein cholesterol, LPS = lipopolysaccharide, $\mathrm{LPL}=$ lipoprotein lipase, $\mathrm{ALP}=$ alkaline phosphatase, $\mathrm{ALT}=$ alanineamino transferase, $\mathrm{AST}=$ aspartate aminotransferase, $\mathrm{MDA}=$ malondialdehyde, $\mathrm{SOD}=$ superoxide dismutase. $\mathrm{Data}$ presented as mean; $n=6$. Means in the same column are significantly different at $\alpha=0.05$. 
Table 7. Effect of supplemental different days of flaxseed on relative gene expression in liver of Peking ducks

\begin{tabular}{llllcccc}
\hline Supplemental days & \multicolumn{1}{c}{ LPIN I } & LPIN 2 & FADS2 & FATP & L-FABP & PPAR- $\alpha$ & WDTCI \\
\hline 30 & $6.94 \pm 1.03^{\mathrm{a}}$ & $1.08 \pm 0.18$ & $1.15 \pm 0.14^{\mathrm{a}}$ & $0.97 \pm 0.13$ & $0.99 \pm 0.22$ & $1.03 \pm 0.10$ & $1.02 \pm 0.20$ \\
20 & $3.41 \pm 0.88^{\mathrm{b}}$ & $1.04 \pm 0.4 \mathrm{I}$ & $0.86 \pm 0.54^{\mathrm{ab}}$ & $0.77 \pm 0.20$ & $0.50 \pm 0.10$ & $0.83 \pm 0.08$ & $1.09 \pm 0.18$ \\
10 & $2.78 \pm 0.89^{\mathrm{b}}$ & $1.53 \pm 0.32$ & $1.02 \pm 0.1^{\mathrm{b}}$ & $0.95 \pm 0.12$ & $0.71 \pm 0.12$ & $0.90 \pm 0.06$ & $1.26 \pm 0.16$ \\
0 & $1.23 \pm 0.39^{\mathrm{b}}$ & $2.30 \pm 0.43$ & $0.56 \pm 0.12^{\mathrm{c}}$ & $0.99 \pm 0.20$ & $0.91 \pm 0.16$ & $0.77 \pm 0.07$ & $1.19 \pm 0.10$ \\
$P$ value & & & & & & \\
ANOVA & $<0.00 \mathrm{I}$ & 0.063 & 0.006 & 0.785 & 0.142 & 0.162 & 0.762 \\
Linear & $<0.00 \mathrm{I}$ & $0.04 \mathrm{I}$ & 0.003 & 0.769 & 0.972 & 0.061 & 0.374 \\
Quadratic & 0.250 & 0.260 & 0.444 & 0.478 & 0.036 & 0.666 & 0.696 \\
\hline
\end{tabular}

Note: $\mathrm{A}=30$ days, $\mathrm{B}=20$ days, $\mathrm{C}=10$ days, $\mathrm{D}=0$ days. LPIN I: lipin I gene encoding lipin I protein; $\mathrm{LPIN}$ 2: lipin 2 gene encoding lipin 2 protein; FADS2 = fatty acid desaturase 2; FATP = fatty acid transport protein; FABP = fatty acid binding protein; PPAR- $\alpha=$ proxisome proliferation activated factor- $\alpha$; WDTCI:WD and tetratricopeptide repeats I. Data are presented as mean; $n=6$. Means in the same column are significantly different at $\alpha=0.05$.

Table 8. Effect of supplemental different days of flaxseed on fatty acid profile of breast muscle of Peking ducks

\begin{tabular}{|c|c|c|c|c|c|c|c|}
\hline Supplemental days & 30 & 20 & 10 & 0 & $P$ & Linear & Quadratic \\
\hline \multicolumn{8}{|l|}{ Fatty acids (mg/l00g) } \\
\hline Myristic acid Cl4:0 & $35.74 \pm 0.39^{d}$ & $45.09 \pm 0.46^{c}$ & $54.30 \pm 1.07^{\mathrm{b}}$ & $85.06 \pm 0.48^{\mathrm{a}}$ & $<0.001$ & $<0.001$ & $<0.001$ \\
\hline palmitic acid $\mathrm{Cl}$ 6:0 & $282.39 \pm 0.66^{d}$ & $295.70 \pm 0.94^{c}$ & $305.52 \pm 0.82^{\mathrm{b}}$ & $366.35 \pm 2.6 \mathrm{I}^{\mathrm{a}}$ & $<0.001$ & $<0.001$ & $<0.001$ \\
\hline Margaric acid CI 7:0 & $49.33 \pm 0.32^{d}$ & $52.65 \pm 0.44^{c}$ & $62.15 \pm 1.82^{b}$ & $86.63 \pm 0.70^{a}$ & $<0.001$ & $<0.001$ & $<0.001$ \\
\hline Total SFA & $367.47 \pm 1.03^{d}$ & $393.45 \pm 0.93^{c}$ & $421.97 \pm 3.18^{b}$ & $538.05 \pm 2.54^{a}$ & $<0.001$ & $<0.001$ & $<0.001$ \\
\hline Palmitoleic acid $\mathrm{Cl6:I}$ & $389.10 \pm 2.10^{d}$ & $399.30 \pm 1.20^{c}$ & $425.39 \pm 1.95^{b}$ & $434.18 \pm 0.89^{a}$ & $<0.001$ & $<0.001$ & 0.667 \\
\hline Oleic acid CI8: $\ln 9 \mathrm{c}$ & $375.38 \pm 3.0 \mathrm{I}^{\mathrm{d}}$ & $413.63 \pm 1.32^{c}$ & $451.15 \pm 0.82^{b}$ & $487.03 \pm 0.83^{a}$ & 0.007 & 0.001 & 0.503 \\
\hline Arachidic acid C20:0 & $25.39 \pm 1.07^{d}$ & $42.79 \pm 0.6 \mathrm{I}^{\mathrm{c}}$ & $50.82 \pm 0.4 \mathrm{I}^{\mathrm{b}}$ & $60.76 \pm 1.18^{a}$ & $<0.001$ & $<0.001$ & $<0.001$ \\
\hline Total MUFA & $789.87 \pm 3.02^{\mathrm{d}}$ & $855.73 \pm 2.42^{c}$ & $927.36 \pm 2.25^{b}$ & $981.96 \pm 1.19^{a}$ & $<0.001$ & $<0.001$ & 0.025 \\
\hline Linoleic acid CI8:2n6 & $426.64 \pm 1.39^{c}$ & $465.41 \pm 2.92^{\mathrm{b}}$ & $487.88 \pm 1.24^{\mathrm{a}}$ & $489.36 \pm 0.96^{\mathrm{a}}$ & $<0.001$ & $<0.001$ & $<0.001$ \\
\hline Ecosadienioc acid C20:2n6 & $15.76 \pm 0.49$ & $16.02 \pm 0.46$ & $15.32 \pm 0.47$ & $15.78 \pm 0.46$ & 0.767 & 0.763 & 0.844 \\
\hline Dihomo- $\gamma$-linolenic acid C20:3n6 & $69.47 \pm 0.5 \mathrm{I}^{\mathrm{b}}$ & $72.76 \pm 0.33^{\mathrm{a}}$ & $74.43 \pm 1.19^{\mathrm{a}}$ & $74.84 \pm 0.53^{\mathrm{a}}$ & $<0.001$ & $<0.001$ & 0.059 \\
\hline Arachidonic acid 20:4n-6 & $10.19 \pm 0.12^{c}$ & $1 \mathrm{I} .64 \pm 0.52^{\mathrm{b}}$ & $12.50 \pm 0.5 \mathrm{I}^{\mathrm{ab}}$ & $12.96 \pm 0.21^{\mathrm{a}}$ & $<0.001$ & $<0.001$ & 0.206 \\
\hline Total n6 & $522.05 \pm 1.5 \mathrm{I}^{\mathrm{c}}$ & $565.85 \pm 3.04^{b}$ & $590.14 \pm 1.89^{a}$ & $592.94 \pm 0.70^{\mathrm{a}}$ & $<0.001$ & $<0.001$ & $<0.001$ \\
\hline Linolenic acid n3 & $339.76 \pm 0.87^{a}$ & $278.53 \pm 2.49^{b}$ & $167.40 \pm 0.33^{c}$ & $35.61 \pm 0.42^{\mathrm{d}}$ & $<0.001$ & $<0.001$ & $<0.001$ \\
\hline ETA C20:4 n3 & $12.44 \pm 0.66^{\mathrm{a}}$ & $8.56 \pm 0.33^{b}$ & $5.37 \pm 0.25^{c}$ & $0.40 \pm 0.03^{d}$ & $<0.001$ & $<0.001$ & 0.180 \\
\hline EPA C20:5 n3 & $18.35 \pm 0.37^{a}$ & $15.93 \pm 0.23^{b}$ & $11.59 \pm 0.25^{c}$ & $2.52 \pm 0.10^{\mathrm{d}}$ & $<0.001$ & $<0.001$ & $<0.001$ \\
\hline DHA C22:6n3 & $15.98 \pm 0.54^{\mathrm{a}}$ & $12.31 \pm 0.52^{\mathrm{b}}$ & $6.17 \pm 0.60^{c}$ & $1.41 \pm 0.14^{\mathrm{d}}$ & $<0.001$ & $<0.001$ & 0.270 \\
\hline Total n3 & $386.54 \pm 1.49^{\mathrm{a}}$ & $315.35 \pm 2.65^{b}$ & $190.52 \pm 0.53^{c}$ & $39.93 \pm 0.47^{d}$ & $<0.001$ & $<0.001$ & $<0.001$ \\
\hline n6:n3 & $1.35 \pm 0.00^{d}$ & $1.79 \pm 0.02^{c}$ & $3.10 \pm 0.01^{b}$ & $14.86 \pm 0.17^{\mathrm{a}}$ & $<0.001$ & $<0.001$ & $<0.001$ \\
\hline
\end{tabular}

Note: $\mathrm{A}=30$ days, $\mathrm{B}=20$ days, $\mathrm{C}=10$ days, $\mathrm{D}=0$ days; $\mathrm{SFA}=$ saturated fatty acid, MUFA = mono-unsaturated fatty acid, ETA = Eicosatetraenoic acid, EPA = Eicosapentaenoic acid, DHA $=$ docosahexaenoic acid; data are presented as mean; $n=6$. Means in the same column are significantly different at $\alpha=0.05$.

(C17:0), total saturated fatty acid (SFA), palmitoleic acid (C16:1), oleic acid (C18:1n9), arachidic acid (C20:0), total MUFA, linoleic acid (C18:2n6), eicosadienoic acid (C20:2 only for skin), dihomo- $\gamma$-linolenic acid (C20:3n6), and total $\mathrm{n}-6$ decreased linearly in the 10,20 , and 30 days flaxseed diet compared to the control base diet $(P<0.05)$. Linolenic acid n-3, ETA (C20:4 n3), EPA (C20:5 n3), DHA $(\mathrm{C} 22: 6 \mathrm{n} 3)$, and total $\mathrm{n}-3$ increased linearly in breast muscle and skin $(P<0.05)$. In breast muscle, DHA increased by $337.58,773.05$, and $1033.33 \%$ and total $n-3$ increased by $377.13,689.75$, and $868.04 \%$ for ducks fed flaxseed for 10,20 , and 30 days, respectively, compared to the control
$(P<0.05)$. In the skin, DHA increased by $658.97,1407.69$, and $2323.08 \%$, while total $\mathrm{n}-3$ increased by $341.74,656.86$, and $852.08 \%$ for ducks fed flaxseed for 10,20 , and 30 days respectively, as compared to the control $(P<0.05)$. The ratio of $n-3$ to $n-6$ (n-6:n-3) decreased linearly in breast muscle and skin compared to control ducks $(P<0.05)$.

\section{Discussion}

Flaxseed is known to be the richest source of $n-3$ fatty acid among the terrestrial sources of n-3 PUFA. It contains seven times more ALA than soybean and corn oil with three times less LA content (35). The marine or preformed sources of n-3 
Table 9. Effect of supplemental different days of flaxseed on fatty acid profile of skin of Peking ducks

\begin{tabular}{|c|c|c|c|c|c|c|c|}
\hline Supplemental days & 30 & 20 & 10 & 0 & $P$ & Linear & Quadratic \\
\hline \multicolumn{8}{|l|}{ Fatty acids (mg//00 g) } \\
\hline Myristic acid Cl4:0 & $38.43 \pm 0.33^{d}$ & $47.27 \pm 0.7 I^{c}$ & $57.98 \pm 1.38^{\mathrm{b}}$ & $87.72 \pm 0.68^{a}$ & $<0.001$ & $<0.001$ & $<0.001$ \\
\hline palmitic acid $\mathrm{Cl}$ 6:0 & $284.82 \pm 0.44^{d}$ & $296.93 \pm 0.92^{c}$ & $308.48 \pm 0.52^{b}$ & $369.61 \pm\left. 1.7\right|^{\mathrm{a}}$ & $<0.001$ & $<0.001$ & $<0.001$ \\
\hline Margaric acid CI7:0 & $50.98 \pm 0.38^{d}$ & $54.84 \pm 0.35^{c}$ & $64.33 \pm 2.08^{\mathrm{b}}$ & $89.51 \pm 0.77^{a}$ & $<0.001$ & $<0.001$ & $<0.001$ \\
\hline Total SFA & $374.24 \pm 0.63^{d}$ & $399.04 \pm 1.09 c$ & $430.79 \pm 3.05^{b}$ & $546.84 \pm 1.50^{\mathrm{a}}$ & $<0.001$ & $<0.001$ & $<0.001$ \\
\hline Palmitoleic acid $\mathrm{Cl} 6$ :I & $395.71 \pm 1.2 \mathrm{I}^{\mathrm{d}}$ & $403.71 \pm 1.52^{c}$ & $429.10 \pm 1.23^{b}$ & $437.50 \pm 0.59^{a}$ & $<0.001$ & $<0.001$ & 0.871 \\
\hline Oleic acid CI8: In9c & $379.14 \pm 2.74^{d}$ & $415.87 \pm 1.43^{c}$ & $456.23 \pm 2.02^{\mathrm{b}}$ & $489.56 \pm 0.70^{\mathrm{a}}$ & $<0.001$ & $<0.001$ & 0.378 \\
\hline Arachidic acid C20:0 & $28.47 \pm 0.89^{d}$ & $46.37 \pm 0.7 I^{c}$ & $53.64 \pm 0.6 \mathrm{I}^{\mathrm{b}}$ & $63.43 \pm 1.66^{\mathrm{a}}$ & $<0.001$ & $<0.001$ & 0.001 \\
\hline Total MUFA & $803.33 \pm 1.83^{d}$ & $865.94 \pm 3.37^{c}$ & $938.97 \pm 2.7 \mathrm{I}^{\mathrm{b}}$ & $990.49 \pm 1.25^{\mathrm{a}}$ & $<0.001$ & $<0.001$ & 0.033 \\
\hline Linoleic acid CI8:2n6 & $446.65 \pm 0.63^{c}$ & $471.53 \pm 0.38^{b c}$ & $479.91 \pm 16.24^{\mathrm{ab}}$ & $505.67 \pm 7.38^{\mathrm{a}}$ & $<0.002$ & $<0.001$ & 0.961 \\
\hline Ecosadienioc acid C20:2n6 & $|3.5| \pm\left. 0.1\right|^{d}$ & $14.52 \pm 0.07^{c}$ & $16.56 \pm 0.09^{b}$ & $18.30 \pm 0.07^{a}$ & $<0.001$ & $<0.001$ & 0.027 \\
\hline Dihomo- $\gamma$-linolenic acid C20:3n6 & $73.73 \pm 0.55^{c}$ & $76.69 \pm 0.59^{b}$ & $80.74 \pm 0.89^{a}$ & $77.95 \pm 0.67^{b}$ & $<0.001$ & $<0.001$ & $<0.001$ \\
\hline Arachidonic acid 20:4n-6 & $10.91 \pm 0.15^{\mathrm{b}}$ & $12.66 \pm 0.07^{\mathrm{a}}$ & $12.98 \pm 0.39 \mathrm{a}$ & $12.42 \pm 0.10^{\mathrm{a}}$ & $<0.001$ & $<0.001$ & $<0.001$ \\
\hline Total n 6 & $544.81 \pm 0.86^{c}$ & $575.4 \mathrm{I} \pm 0.44^{\mathrm{b}}$ & $590.19 \pm 16.36^{\mathrm{ab}}$ & $614.33 \pm 7.84^{a}$ & $<0.001$ & $<0.001$ & 0.726 \\
\hline Linolenic acid n3 & $358.78 \pm 3.43^{\mathrm{a}}$ & $288.15 \pm 2.18^{b}$ & $168.88 \pm\left. 2.8\right|^{c}$ & $40.36 \pm 0.21^{d}$ & $<0.001$ & $<0.001$ & $<0.001$ \\
\hline ETA C20:4 n3 & $11.94 \pm 0.34^{\mathrm{a}}$ & $7.06 \pm 0.26^{b}$ & $4.19 \pm 0.35^{c}$ & $0.45 \pm 0.04^{d}$ & $<0.001$ & $<0.001$ & 0.053 \\
\hline EPA C20:5 n3 & $19.10 \pm 0.47^{a}$ & $17.94 \pm 0.32^{\mathrm{b}}$ & $10.65 \pm 0.3 I^{c}$ & $1.34 \pm 0.05^{d}$ & $<0.001$ & $<0.001$ & $<0.001$ \\
\hline DHA C22:6 n3 & $18.90 \pm 0.48^{\mathrm{a}}$ & $11.76 \pm 1.98^{b}$ & $5.92 \pm 0.32^{c}$ & $0.78 \pm 0.04^{d}$ & $<0.001$ & $<0.001$ & 0.343 \\
\hline Total n3 & $408.73 \pm 2.77^{a}$ & $324.92 \pm 3.60^{\mathrm{b}}$ & $189.64 \pm 3.48^{c}$ & $42.93 \pm 0.24^{d}$ & $<0.001$ & $<0.001$ & $<0.001$ \\
\hline n6:n3 & $\mathrm{I} .33 \pm 0.0 \mathrm{I}^{\mathrm{d}}$ & $1.77 \pm 0.02^{c}$ & $3.11 \pm 0.03^{b}$ & $|4.3| \pm\left. 0.1\right|^{a}$ & $<0.001$ & $<0.001$ & $<0.001$ \\
\hline
\end{tabular}

Note: $\mathrm{A}=30$ days, $\mathrm{B}=20$ days, $\mathrm{C}=10$ days, $\mathrm{D}=0$ days. SFA $=$ saturated fatty acid, MUFA = mono-unsaturated fatty acid, ETA $=$ Eicosatetraenoic acid, $\mathrm{EPA}=$ Eicosapentaenoic Acid, $\mathrm{DHA}=$ docosahexaenoic acid.Data presented as mean; $n=6$. Means in the same column are significantly different at $\alpha=0.05$.

are fish oil and microalgae and their supplementation increase DHA in the eggs threefold as compared to flaxseed oil but it gives fishy flavor to the products $(36,37)$. Flaxseed supplementation in ducks increased the number of bacteria causing inflammation but this was diminished with prolonged duration of flaxseed feeding (38). Previous reports have shown the poor growth performance of birds fed with flaxseed (20). In this study, ducks' growth performance was negatively affected when fed with flaxseed at $10 \%$ for 30 days, most likely due to the presence of anti-nutritional factors such as linatine and non-starch polysaccharides (NSP) in flaxseed mucilage (39). This is because poultry lack enzymes to break the bonds between sugars in NSP (40), which could lower the digestion and availability of nutrients (41). Hence, the use of enzymes can be helpful to degrade NSP and alleviate the negative effect of flaxseed on growth performance in poultry (19). Previous studies report that increased LPL is associated with reduced fat accumulation in the body by regulating endogenous fatty acid oxidation which could reduce body weight $(42,43)$. Moreover, LIPIN-1 mRNA expression in chicken has also shown to decrease fat deposition (44). In this study, flaxseed increased the LPL and LIPIN-1 gene expression which could reduce the abdominal fat deposition and could ultimately be responsible for reduced body weight gain in the ducks.

The relative weight of skin and abdominal fat decreased linearly with increasing duration of the flaxseed diet in agreement with previous results of linseed oil fed broilers
$(6,20)$. In our study, increasing the duration of the flaxseed diet resulted in a linear increase of good cholesterol (HDL) and a linear decrease in bad cholesterol like LDL, VLDL, and TG. This is in agreement with results indicating that diet enrichment with PUFA has a beneficial effect on serum lipid profile (45) or that increasing n-3 PUFA in the diet of chickens increases HDL and reduces TG (46). This reduction may be related to the role of n-3 FA in the suppression of TG, apolipoprotein synthesis, higher elimination of VLDL by peripheral tissues of the liver and higher excretion of bile via feces (47), resulting in a reduction in cholesterol and TG concentrations in serum.

High-fat diets increase the serum LPS activity by increasing permeability of the intestine through inhibition of the protein expression from the tight junctions, allowing LPS molecules to enter from the intestine into circulation (48-50). In our study, LPS activity increased with the duration of the flaxseed diet. It is believed that HDL helps in LPS detoxification but its binding with HDL makes it difficult to clear from circulation (51). In our study, LPL enzymatic activity increased linearly with the duration of the flaxseed diet. LPL activity in animal tissues increases with the consumption of diets rich in PUFA (52). The enzymatic activity of LPL represents a limiting step for the entry of dietary fatty acid into tissues because LPL hydrolyzes circulating TG in the form of porto microns and verylow-density lipoproteins in order to enter the tissues (53). 
Fatty acid manipulation via dietary means may provide an effective way to obtain n-3 enriched animal products for human consumption. Flaxseed oil and full-fat flaxseed are valuable sources of ALA in chicken diets and effectively incorporated from feed to the bird's tissues (54). Dietary flaxseed oil significantly increased n-3 PUFA concentrations and decreased n-6:n-3 PUFA ratios in birds fed diets containing soybean oil (55). In our study, total n3 increased linearly in breast muscle and skin, while SFA, MUFA, and n-6 PUFA linearly decreased with the duration of the flaxseed diet.

In poultry, de novo lipogenesis occurs in the liver from dietary carbohydrates. These reactions are catalyzed by glucose-6-phosphate, dehydrogenase, and malic enzyme (56). The conversion of oil sources rich in C18:3 into $\mathrm{C} 20: 5$ and $\mathrm{C} 22: 6$ had been reported previously (57). However, in our experiment, $\mathrm{n}-3$ content in breast muscles and skin increased with the duration of the diet. C20:5 and C22:6 FA were observed in breast and skin, indicating hepatic elongation and desaturation of FA. The increase in omega-3 in breast muscle and its conversion to EPA and DHA with increased duration of the diet might be due to the higher hepatic expression of the FADS2 gene, which competes for both n-3 and n-6 (7).

The liver is the major site of lipogenesis and de novo fatty acid biosynthesis as the liver transfers lipids to other tissues through blood and exerts an effect on the lipid content and composition (58). LPIN1, LPIN2, WDTC1, and FADS2, are lipid metabolism genes with vital functions in fat metabolism (6). LPIN1 is an enzyme, involved in the de novo biosynthesis of TG and its mRNA is abundant in adipose tissue and muscle tissue of mice (59). In our study, feeding flaxseed to ducks for 30 days upregulated mRNA expression of LPIN1 (10). Lipin1 can also act as a transcriptional co-activator in relation to peroxisome proliferator-activated receptor alpha (PPAR- $\alpha$ ). These are nuclear receptors that act as major regulators of hepatic lipid catabolism, targeting gene expression associated with inflammation, glucose metabolism, and most importantly, the lipid-related pathway (60). Lipin-1 enhanced expression of PPAR- $\alpha$ target genes with direct interaction with PPAR receptors (61). In our study, increased expression of Lipin-1 activated PPAR- $\alpha$ expression, but not significantly, indicating that Lipin-1 can co-activate PPAR- $\alpha$.

FADS2 expression increased at 10 and 30 days while at 20 days its expression was still above that of the control diet. A previous study (28) also reported that flaxseed oil increased expression of FADS2 at day 7 of feeding prior to slaughter, but FADS2 decreased when the duration of the flaxseed diet increased. Jing et al. (9) suggested that FADS2 gene expression changed over time according to the dietary n-6:n-3 ratio, supporting the concept that metabolic enzymes of long-chain PUFA are modulated by dietary fatty acid composition. It was proposed in the previous studies that consumption of an n-3 enriched diet could stimulate mRNA expression of FADS2, due to the higher affinity of FADS2 for n-3 than for n-6 $(62,63)$.

WDTC1 is an obesity-linked gene which appears to inhibit fat formation in a dosage-sensitive manner in both animals and humans (64). In this study, feeding flaxseed had no effect on WDTC1 gene expression. FATP, proposed as a major FA transporter in intestinal lipid absorption, is necessary for the transportation of long-chain FA across the plasma membrane and for esterification (5). L-FABP played a key role in transporting FA through the cytosol of absorptive cells (65). Long-chain polyunsaturated FA had a higher affinity towards L-FABP (66). In our study, there was no difference in expression of L-FATP and FABP between control and flaxseed fed ducks, which could be due to the high level of n-6 PUFA in the control group diets and breast muscle. Over-expression of LPIN1 promotes fat deposition in mice (44) but decreases fat deposition in chicken (67). In addition, high hepatic PPAR- $\alpha$ expression reduced abdominal fat accumulation in broilers (68). We found that an increase in LPIN 1 gene expression reduced abdominal fat deposition and that PPAR- $\alpha$ expression did not change significantly but was higher after 30 days of feeding flaxseed, compared to the control diet, resulting in reduced abdominal fat in the Peking ducks.

In our study, the observed decrease in the lipid oxidation in the meat, liver, and jejunal mucosa, might be related to the antioxidant activity of vitamin $\mathrm{E}$ in the premix added to the diets. The contents of vitamin $\mathrm{E}$ in the body could enhance total antioxidant activity. AST and, especially, ALT, are good indicators of liver damage. High levels of liver enzymes in serum are usually due to hepatocytes necrosis and altered membrane permeability (69). In the current study, flaxseed with vitamin E decreased hepatic enzymatic activity like AST in serum. The improvement in serum's AST could be due to the beneficial effect of vitamin $\mathrm{E}$ on duck liver. The drip loss (water-holding-capacity) of meat depends on intramuscular lipids, moisture content, and lipid peroxide content in the muscle (70). Flaxseed supplementation resulted in an increase in the oxidation of breast meat (71). We found that the drip loss of breast meat decreased with the duration of the flaxseed diet. This improvement in drip loss might be due to the antioxidant capacity of vitamin E, which could lower the lipid oxidation in the breast muscle, enhance antioxidant enzymes' activities, enhance the integrity of cellular membranes, and thereby decrease drip loss in the muscle (72).

From the economic perspective, the price of flaxseed is approximately twice the price of wheat and corn. In order, to get maximum profit from the supplemented flaxseed feeding duration to get duck meat enriched with n-3 PUFA, a threshold level is required for labeling this 
meat as n-3 enriched duck meat. According to the Canadian Food Inspection Agency, 2003, the threshold level of $300 \mathrm{mg} / 100 \mathrm{~g}$ of total $\mathrm{n}-3$ fatty acid of meat is required for labeling it as n-3 FA enriched (73). However, in this study, increasing the duration of feeding a flaxseed diet had mildly decreased BW and increased FCR which ultimately elevated the cost of production. On the other hand, feeding flaxseed for the 20 days' duration potentially enriched the duck breast muscle with n-3 PUFA (386.54 mg/100 g of meat) before the completion of the flaxseed-feeding duration (30 days). A feeding duration of 20 days before slaughtering is optimal to attain a threshold level of n-3 PUFA in the Peking duck meat which would lower the cost of production for commercial use. It is worthy of attention to note that n-3 enriched meat and eggs are already popular in the world, and health-conscious consumers are willing to pay a premium price for them (74). Use of carbohydrase enzyme may reduce the negative effect of flaxseed in poultry (75), which could increase the palatability of flaxseed and also improve the n-3 enrichment efficiency for health-conscious consumers.

\section{Conclusion}

Feeding flaxseed with vitamin $\mathrm{E}$ for a period of 30 days had a mild adverse effect on the performance of ducks but enhanced meat quality and decreased lipid oxidation in meat. The feeding period of 20 days before slaughtering is adequate to enrich meat with EPA and DHA and decrease the n- 6 to $n-3$ ratio. The enrichment of duck meat with long-chain PUFA (EPA and DHA) by using flaxseed is appropriate for health-conscious consumers and could replace the marine sources of EPA and DHA in the diet of poultry. Further studies are suggested to investigate the effect of flaxseed on the fatty acid profile of raw and cooked poultry meat.

\section{Acknowledgements}

This research was financially supported by Beijing Technology Program [Z181100009318008], and Beijing Innovation Research Team of Modern Agriculture [BAIC04-2019]. The funder had no role in study design, data collection, analysis, decision to publish, and preparation of manuscript.

\section{Conflict of interest and funding}

The authors declare no potential conflict of interest.

\section{References}

1. Saini RK, Keum YS. Omega-3 and omega-6 polyunsaturated fatty acids: dietary sources, metabolism, and significance - a review. Life Sci 2018; 203: 255-67. doi: 10.1074/jbc.M401231200.
2. Desnoyers M, Gilbert K, Rousseau G. Cardioprotective effects of omega-3 polyunsaturated fatty acids: dichotomy between experimental and clinical studies. Marine Drugs 2018; 16: 234. doi: 10.3390/md16070234.

3. Nguyen DV, Malau-Aduli BS, Cavalieri J, Nichols PD, Malau-Aduli AE. Supplementation with plant-derived oils rich in omega-3 polyunsaturated fatty acids for lamb production. Vet Anim Sci 2018; 6: 29-40. doi: 10.1016/j.vas.2018.08.001.

4. Richieri GV, Ogata RT, Kleinfeld AM. Equilibrium constants for the binding of fatty acids with fatty acid-binding proteins from adipocyte, intestine, heart, and liver measured with the fluorescent probe ADIFAB. J Biol Chem 1994; 269: 23918-30.

5. Coe NR, Smith AJ, Frohnert BI, Watkins PA, Bernlohr DA. The fatty acid transport protein (FATP1) is a very long chain acyl-coasynthetase. J Biol Chem 1999; 274: 36300-4. doi: 10.1074/jbc.274.51.36300.

6. Chen W, Zhao R, Yan BX, Zhang JS, Huang YQ, Wang ZX, et al. Effects of the replacement of corn oil with linseed oil on fatty acid composition and the expression of lipogenic genes in broiler chickens. Czech J Anim Sci 2014; 59: 353-64. doi: 10.17221/7586-CJAS

7. Guillou H, D’Andrea S, Rioux V, Jan S, Legrand P. The surprising diversity of delta 6-desaturase substrates. Biochem Soc Trans 2004; 32: 86-7. doi: 10.1042/BST0320086.

8. Kersten S. Integrated physiology and systems biology of PPAR $\alpha$. Mol Metabol 2014; 3: 354-71. doi: 10.1016/j. molmet.2014.02.002.

9. Jing M, Gakhar N, Gibson RA, House JD. Dietary and ontogenic regulation of fatty acid desaturase and elongase expression in broiler chickens. Prostaglandins Leukot Essent Fatty Acids 2013; 89: 107-13. doi: 10.1016/j.plefa.2013.05.006.

10. Yan BX, Zhao R, Wang JP, ChenW, Huang YQ, Wang ZX, et al. Effect of different dietary oil sources on the growth performance, blood characteristics, fatty acid profiles, and expression of lipogenic genes in the liver of broiler chickens. Czech J Anim Sci 2015; 60: 487-97. doi: 10.17221/8557-CJAS.

11. Patsch JR, Prasad S, Gotto JAM, Patsch W. High density lipoprotein2. Relationship of the plasma levels of this lipoprotein species to its composition, to the magnitude of postprandial lipemia, and to the activities of lipoprotein lipase and hepatic lipase. J Clin Invest 1987; 80: 341-7. doi: 10.1172/JCI113078

12. Mueller M, Lindner B, Kusumoto S, Fukase K, Schromm AB, Seydel U. Aggregates are the biologically active units of endotoxin. J Biol Chem 2004; 279: 26307-13. doi: 10.1074/jbc. M401231200

13. Kaufman RL, Matson CF, Beisel WR.. Hypertriglyceridemia produced by endotoxIn: role of impaired triglyceride disposal mechanisms. J Infect Dis 1976; 133: 548-55. doi: 10.1093/ infdis/133.5.548

14. Shim YY, Gui B, Arnison PG, Wang Y, Reaney MJ. Flaxseed (Linum usitatissimum $\mathrm{L}$.) bioactive compounds and peptide nomenclature: a review. Trends Food Sci Technol 2014; 38: 5-20. doi: 10.1016/j.tifs.2014.03.011.

15. de-Moura CMA, Junior MSS, Fiorda FA, Caliari M, Vera R, Grossmann MVE. Cooking and texture properties of gluten-free fettuccine processed from defatted flaxseed flour and rice flour. Int J Food Sci Technol 2016; 51: 1495-501. doi: 10.1111/ijfs. 13097.

16. Parikh M, Maddaford TG, Austria, JA, Aliani M, Netticadan T, Pierce GN. Dietary flaxseed as a strategy or improving human health. Nutrients 2019; 11: 1171. doi: 10.3390/nu11051171

17. Priy R, Katoch M, Kumar A, Csk V, Pradesh H, Vishwavidyalya K. Flaxseed-composition and its health benefits. Res Environ Life Sci 2017; 9: 310-16. doi: 10.13140/RG.2.2.35208.93448. 
18. Mattila PH, Pihlava JM, Hellström J, Nurmi M, Eurola M, Mäkinen, S, et al. Contents of phytochemicals and antinutritional factors in commercial protein-rich plant products. Food Quality Safety 2018; 2: 213-19. doi: 10.1093/fqsafe/fyy021.

19. Jankowski J, Zdunczyk Z, Mikulski D, Naczmanski J, Juskiewicz J, Troszynska A, et al. Inclusion of flaxseed in turkey diets decreases the $n-6 / n-3$ PUFA ratio and increases the proportion of biologically active EPA and DHA without affecting meat quality. Eur J Lipid Sci Technol 2015; 117: 797-809. doi: 10.1002/ejlt.201400186.

20. Mridula D, Kaur D, Nagra SS, Barnwal P, Gurumayum S, Singh KK. Growth performance and quality characteristics of flaxseed-fed broiler chicks. J Appl Anim Res 2015; 43: 345-51. doi: 10.1080/09712119.2014.978773.

21. Salami SA, Majokaa MA, Saha S, Garbera A, Gabarroua JF. Efficacy of dietary antioxidants on broiler oxidative stress, performance and meat quality: science and market. Avian Biol Res 2015; 8 :65-78. doi: 10.3184/175815515X14291701859483.

22. Anjum FM, Muhammad FH, Muhammad IK, Muhammad S, Muhammad SA. Impact of extruded flaxseed meal supplemented diet on growth performance, oxidative stability and quality of broiler meat and meat products. Lipids Health Dis 2013; 12: 13. doi: 10.1186/1476-511X-12-13.

23. Farag MR, Mahmoud A, Kuldeep D. Flaxseed oil alleviates toxic effects of subacute exposure to acephate on liver and kidney of broiler chicks. Asian J Anim Vet Adv 2017; 12: 61-70. doi: 10.3923/ajava.2017.61.70.

24. Barroeta AC. Nutritive value of poultry meat: relationship between vitamin E and PUFA. World Poult Sci J 2007; 63: 277-84. doi: $10.1017 /$ S0043933907001468.

25. Leskovec J, Levart A, Nemec Svete A, Peric L, Dukic Stojcic $\mathrm{M}$, Zikic D, et al. Effects of supplementation with $\alpha$-tocopherol, ascorbic acid, selenium, or their combination in linseed oilenriched diets on the oxidative status in broilers. Poult Sci 2018; 97: 1641-50. doi: 10.3382/ps/pey004.

26. DiNicolantonio JJ, O'Keefe JH. Omega-6 vegetable oils as a driver of coronary heart disease: the oxidized linoleic acid hypothesis. Open Heart 2018; 5:e000898. doi: 10.1136/ openhrt-2018-000898

27. Huang FR, Zhan ZP, Luo J, Liu ZX, Peng J. Duration of dietary linseed feeding affects the intramuscular fat, muscle mass and fatty acid composition in pig muscle. Livestock Sci 2008; 118: 132-9. doi: 10.1016/j.livsci.2008.01.002.

28. Mirshekar R, Boldaji F, Dastar B, Yamchi A, Pashaei S. Longer consumption of flaxseed oil enhances n-3 fatty acid content of chicken meat and expression of FADS2 gene. Eur J Lipid Sci Technol 2015; 117: 810-19. doi: 10.1002/ejlt.201300500.

29. The Ministry of Agricultural of the People's Republic of China. 2004. Chinese performance terms and measurement for poultry (NY/T 823-2004).

30. Mikulski D, Jankowski J, Zdunczyk Z, Juskiewicz J, Slominski BA. The effect of different dietary levels of rapeseed meal on growth performance, carcass traits, and meat quality in turkeys. Poult Sci 2012; 91: 215-23. doi: 10.3382/ ps.2011-01587.

31. Huang L, Ma XY, Jiang ZY, Hu YJ, Zheng CT, Yang XF, et al. Effects of soybean isoflavone on intestinal antioxidant capacity and cytokines in young piglets fed oxidized fish oil. J Zhejiang Univ-Sci B (Biomed \& Biotechnol) 2016; 17: 965-74. doi: 10.1631/jzus.B1600078.

32. Livak KJ, Schmittgen TD. Analysis of relative gene expression data using real-time quantitative pcr and the 2(-delta deltac $(\mathrm{t})$ method. Methods 2001; 25: 402-8. doi: 10.1006/meth.2001.1262.
33. Christie WW. Preparation of ester derivatives of fatty acids for chromatographic analysis. Adv Lipid Methodol 1993; 2: 69-111.

34. SPSS Software. SPSS Base 20.0. Chicago, IL: SPSS Inc; 2010.

35. National Research Council Subcommittee on Poultry Nutrition. Nutrient requirements of poultry. 9th revised ed. Washington, DC: National Academy Press; 1994. doi: 10.17226/2114.

36. Lee SA, Whenham N, Bedford MR. Review on docosahexaenoic acid in poultry and swine nutrition: consequence of enriched animal products on performance and health characteristics. Anim Nutr 2019; 5: 11-21. doi: 10.1016/j.aninu.2018.09.001.

37. Neijat M, Ojekudo O, House JD. Effect of flaxseed oil and microalgae DHA on the production performance, fatty acids and total lipids of egg yolk and plasma in laying hens. Prostaglandins Leukot Essent Fatty Acids 2016; 115, 77-88. doi: 10.1016/j. plefa.2016.10.010.

38. Wu Y, Wang Y, Yin D, Shahid MS, Yuan J. Flaxseed diet caused inflammation by altering the gut microbiota of Peking ducks. Anim Biotechnol 2019; 30: 1-12. doi: 10.1080/10495398.2019.1634579.

39. Klosterman HJ, Lamoureux GL, Parsons JL. Isolation, characterization and synthesis of linatine. A. vitamin B6 antagonist from flaxseed (Linum usitatissimum). Biochemistry 1967; 6: 170-7. doi: 10.1021/bi00853a028.

40. Classen HL, Bedford MR. The use of enzymes to improve the nutritive value of poultry feeds. In: Haresign W, Cole DJA, eds. Recent advances in animal nutrition. Oxford, UK: Butterworth-Heinemann LTD; 1991, pp. 95-116.

41. Rebole A, Rodriguez ML, Ortiz LT, Alzueta C, Centeno C, Trevino J. Mucilage in linseed: effects on the intestinal viscosity and nutrient digestion in broiler chicks. J Sci Food Agri 2002; 82: 1171-6. doi: 10.1002/jsfa.1164.

42. Schwartz RS, Brunzell JD. Increase of adipose tissue lipoprotein lipase activity with weight loss. J Clin Invest 1981; 67: 1425-30. doi: 10.1172/JCI110171

43. Fukumitsu S, Aida K, UenoN, Ozawa S, Takahashi Y, Kobori M. Flaxseed lignan attenuates high-fat diet-induced fat accumulation and induces adiponectin expression in mice. Br J Nutr 2008; 100: 669-76. doi: 10.1017/S0007114508911570.

44. Wang XK, Chen W, Huang YQ, Kang XT, Wang JP, Li GX, et al. Identification of the transcript isoforms and expression characteristics for chicken Lpin1. Animal 2012; 6: 1897-190. doi: $10.1017 /$ S1751731112001358.

45. Du M, Ahn DU. Dietary CLA affects lipid metabolism in broiler chicks. Lipids 2003; 38: 505-11. doi: 10.1007/s11745-003-1091-z.

46. Ibrahim D, El-Sayed R, Khater SI, Said EN, El-Mandrawy SAM. Changing dietary n-6:n-3 ratio using different oil sources affects performance, behavior, cytokines mRNA expression and meat fatty acid profile of broiler chickens. Anim Nutri 2017; 4: 44-51. doi: 10.1016/j.aninu.2017.08.003.

47. Leaf A, Weber PC. Cardiovascular effects of n-3 fatty acids. N Engl J Med 1988; 318: 549-57. doi: 10.1056/ NEJM198803033180905.

48. Erridge C, Attina T, Spickett CM, Webb DJ. A high-fat meal induces low grade endotoxemia: evidence of a novel mechanism of postprandial inflammation. Am J Clin Nutr 2007; 86: 1286-92. doi: 10.1093/ajen/86.5.1286.

49. Cani PD, Bibiloni R, Knauf C, Waget A, Neyrinck AM, Delzenne NM, et al. Changes in gut microbiota control metabolic endotoxemia-induced inflammation in high-fat diet induced obesity and diabetes in mice. Diabetes 2008; 57: 1470-81. doi: $10.2337 / \mathrm{db} 07-1403$.

50. Caesar R, Fak F, Backhed F. Effects of gut microbiota on obesity and atherosclerosis via modulation of inflammation 
and lipid metabolism. J Intern Med 2010; 268: 320-8. doi: 10.1111/j.1365-2796.2010.02270.x.

51. Birjmohun RS, Van-Leuven SI, Levels JHM, van't Veer C, Kuivenhoven JA, Meijers JCM, et al. High-density lipoprotein attenuates inflammation and coagulation response on endotoxin challenge in humans. Arteriosclr Thromb Vasc Biol 2007; 27: 1153-8. doi: 10.1161/ATVBAHA.106.136325.

52. Ferrini G, Manzanilla E, Menoyo D, Esteve-Garcia E, Baucells M, Barroeta A. Effects of dietary n-3 fatty acids in fat metabolism and thyroid hormone levels when compared to dietary saturated fatty acids in chickens. Livestock Sci 2010; 131: 287-91. doi: 10.1016/j.livsci.2010.03.017.

53. Bensadoun A. Lipoprotein-lipase. Annu Rev Nutr 1991; 11: 217-37. doi: 10.1146/annurev.nu.11.070191.001245.

54. Abbasi F, Samadi F, Jafari SM, Ramezanpour S, Shargh MS. Ultrasound-assisted preparation of flaxseed oil nanoemulsions coated with alginate-whey protein for targeted delivery of omega-3 fatty acids into the lower sections of gastrointestinal tract to enrich broiler meat. Ultrason Sonochem 2019; 50: 208-17. doi: 10.1016/j.ultsonch.2018.09.014.

55. Jankowski J, Zdunczyk Z, Mikulski D, Juskiewicz J, Naczmanski J, Pomianowski JF, et al. Fatty acid profile, oxidative stability, and sensory properties of breast meat from turkeys fed diets with a different n-6/n-3 PUFA ratio. Eur J Lipid Sci Technol 2012; 114: 1025-35. doi: 10.1002/ejlt.201200003.

56. Alvarez M, Diez JA, Lopez-Bote CJ, Gallego M, Bautista JM. Short term modulation of lipogenesis by macronutrients in rainbow trout (Oncorhynchus mykiss) hepatocytes. Br J Nutr 2000; 84: 619-28. doi: 10.1017/S0007114500001951.

57. Sealls W, Gonzalez M, Brosnan MJ, Black PN, Dirusso CC. Dietary polyunsaturated fatty acids (C18:2 omega 6 and C18:3 omega 3) do not suppress hepatic lipogenesis. Biochim Biophys Acta 2008; 1781: 406-14. doi: 10.1016/j.bbalip.2008.06.010.

58. Lodhi IJ, Wei X, Semenkovich CF. Lipoexpediency: de novo lipogenesis as a metabolic signal transmitter. Trends Endocrinol Metabol 2011; 22: 1-8. doi: 10.1016/j.tem.2010.09.002.

59. Donkor J, Sariahmetoglu M, Dewald J, Brindley DN, Reue K. Three mammalian lipins act as phosphatidate phosphatases with distinct tissue expression patterns. J Biol Chem 2007; 282: 3450-7. doi: 10.1074/jbc.M610745200.

60. Bionaz M, Chen S, Khan MJ, Loor JJ. Functional role of PPARs in ruminants: potential targets for fine-tuning metabolism during growth and lactation. PPAR Res 2013; 2013: 28. doi: $10.1155 / 2013 / 684159$.

61. Finck BN, Gropler MC, Chen Z, Leone TC, Croce MA, Harris TE, et al. Lipin 1 is an inducible amplifier of the hepatic PGC-1 $\alpha /$ $\operatorname{PPAR} \alpha$ regulatory pathway. Cell Metab 2006; 4: 199-210. doi: 10.1016/j.cmet.2006.08.005.

62. Poureslami R, Reas K, Turchini GM, Huyghebaert G, De SS. Effect of diet, sex and age on fatty acid metabolism in broiler chickens: n-3 and n-6 PUFA. Br J Nutr 2010; 104: 189-97. doi: 10.1017/S0007114510000395.

63. Candela GC, Lopez LMB, Kohen VL. Importance of a balanced omega6/omega 3 ratio for the maintenance of health: nutritional recommendations. Nutr Hosp 2011; 26: 323-329. doi: 10.3305/ nh.2011.26.2.5117.

64. Suh JM, Zeve D, McKay R, Seo J, Salo Z, Li R, et al. Adipose is a conserved dosage-sensitive antiobesity gene. Cell Metabol 2007; 6: 195-207. doi: 10.1016/j.cmet.2007.08.001.

65. Krogdahl A. Digestion and absorption of lipids in poultry. J Nutr 1985; 115: 675-85. doi: 10.1093/jn/115.5.675.

66. Richieri GV, Ogata RT, Kleinfeld AM. Fatty acid interactions with native and mutant fatty acid binding protein. Mol Cell Biochem 1999; 192: 77-85. doi: 10.1023/A: 1006826405151.

67. Phan J, Reue K. Lipin, a lipodystrophy and obesity gene. Cell Metabol 2005; 1: 73-83. doi: 10.1016/j.cmet.2004.12.002.

68. Han J, Li L, Wang D, Ma H. (-)-Hydroxycitric acid reduced fat deposition via regulating lipid metabolism-related gene expression in broiler chickens. Lipids Health Dis 2016; 15: 37. doi: 10.1186/s12944-016-0208-5.

69. Amiridumari H, Sarir H, Afzali N, Fanimakki O. Effects of milk thistle seed against aflatoxin B1 in broiler model. J Res Med Sci 2013; 18: 786-90.

70. Cao FL, Zhang XH, Yu WW, Zhao LG, Wang T. Effect of feeding fermented Ginkgo biloba leaves on growth performance, meat quality, and lipid metabolism in broilers. Poult Sci 2012; 91: 1210-21. doi: 10.3382/ps.2011-01886.

71. Huang JF, Huang CC, Lai MK, Lin JH, Lee CH, Wang TY. Effects of dietary fish oil on the contents of eicosapentaenoic acid and docosahexaenoic acid and sensory evaluation of the breast meat in mule ducks. Asian Aust J Anim Sci 2006; 19: 231-5. doi: 10.5713/ajas.2006.231.

72. Kobus-Cisowska J, Flaczyk E, Rudzińska M, Kmiecik D. Antioxidant properties of extracts from Ginkgo biloba leaves in meatballs. Meat Sci 2014; 97: 174-80. doi: 10.1016/j. meatsci.2014.01.011.

73. Canadian Food Inspection Agency. 2003 Guide to food labelling and advertising. Available from: http://www.inspection.gc.ca/ english/fssa/labeti/guide/ch7be.shtml\#7 [cited 19 April 2008].

74. Bhalerao S, Hegde M, Katyare S, Kadam S. Promotion of omega-3 chicken meat production: an Indian perspective. Worlds Poult Sci J. 2014; 70: 365-74. doi: 10.1017/S004393 3914000373.

75. Behesti-Moghadam MH, Shehab A, Cherian G. Production performance, quality and lipid composition of eggs from laying hens fed heated flaxseed with carbohydrase enzymes. J Appl Poult Res 2019; 0: 1-9. In press. doi: 10.3382/japr/pfz034.

\footnotetext{
*Jianmin Yuan

State Key

Laboratory of Animal Nutrition,

College of Animal Science and Technology,

China Agricultural University,

Beijing 100193, P. R. China

Email:yuanjm@cau.edu.cn
} 